\title{
Does Adequate Lumbar Segmental Motion Reflect Recovery Process in Acute Lumbar Disc Herniation?
}

\author{
Hyuntae Kim, MD, Ho Joong Jung, MD, Minsun Kim, MD, \\ Seong-Eun Koh, MD, PhD, In-Sik Lee, MD, PhD
}

Department of Rehabilitation Medicine, Konkuk University School of Medicine \& Konkuk University Medical Center, Seoul, Korea

Objective To investigate if the extent of lumbar segmental motion is correlated with the recovery process in the form of pain intensity in patients with acute single level lumbar disc herniation (LDH).

Methods A retrospective review of medical records was performed on patients presented with acute low back pain from January 2011 to December 2017. With prerequisites of undergoing both lumbar spine magnetic resonance imaging and functional radiography, patients with etiologies other than single level LDH were excluded. A total of 46 patients were selected, including 27 patients with disc herniation at L4-5 level and 19 patients at L5-S1 level. Pearson correlation analysis of pain intensity against segmental range of motion (sROM) and percentage of sROM of each lumbar segment was performed at the initial evaluation point and follow-ups.

Results Serial documentation of pain intensity and functional radiography exhibited an inverse correlation between changes in visual analogue scale (VAS) and sROM in single level LDH at L4-5 level $(\mathrm{r}=-0.69, \mathrm{p}<0.05)$. In addition, percentage of sROM showed a negative correlation with pain intensity at the aforementioned segment $(\mathrm{r}=-0.74, \mathrm{p}<0.05)$. Initial pain intensity was also inversely correlated to sROM of the affected segment $(\mathrm{r}=-0.83$, $\mathrm{p}<0.01$ at L4-5; $\mathrm{r}=-0.82, \mathrm{p}<0.05$ at L5-S1).

Conclusion Improvement in sagittal mobility of the affected segment in LDH adequately reflected mitigation of low back pain during the recovery process. This conjunction could illustrate that the involved segment is overcoming natural immobilization, evidently demonstrating an inverse relationship between initial pain intensity and limitation of sagittal range of motion.

Keywords Low back pain, Intervertebral disc displacement, Range of Motion, Articular

Department of Rehabilitation Medicine, Konkuk University Medical Center, 120-1 Neungdong-ro, Gwangjin-gu, Seoul 05030, Korea. Tel: +82-2-20305347, Fax: +82-2-2030-5379, E-mail: mdlis@kuh.ac.kr

ORCID: Hyuntae Kim (http://orcid.org/0000-0001-9230-9977); Ho Joong Jung (http://orcid.org/0000-0001-7066-3369); Minsun Kim (http://orcid. org/0000-0002-4861-1762); Seong-Eun Koh (http://orcid.org/0000-0002-0446-1889); In-Sik Lee (http://orcid.org/0000-0001-8167-7168).

@ This is an open-access article distributed under the terms of the Creative Commons Attribution Non-Commercial License (http://creativecommons.org/ licenses/by-nc/4.0) which permits unrestricted noncommercial use, distribution, and reproduction in any medium, provided the original work is properly cited. Copyright $\odot 2019$ by Korean Academy of Rehabilitation Medicine 


\section{INTRODUCTION}

Resuming work or activities of daily living after suffering an episode of acute lumbar disc herniation (LDH) can be difficult. That is not only in its literal sense that disc herniation itself may be physically gruesome, but also in its figurative sense in determining when patients should return to premorbid level of physical activities. While the prevalence of herniated disc disease has been estimated to be $2 \%-3 \%[1,2]$ which varies depending on age and gender, different extent of disc herniation and their relative lack of correlation with clinical symptoms render it hard for clinicians to gauge how to make recommendations in terms of when to return to physical activities. Given that enormous economical toll is associated with lumbar disc disorders [3], especially in lost work time and reduced productivity, constructing proper strategies to reinstitute premorbid level of activity while establishing necessary preventive measures against recurrent episodes appears to be critical.

In patients with acute low back pain, restriction of activities has been conventionally considered as part of a treatment protocol. However, the duration and stringency for such restriction after a lumbosacral disc herniation remains controversial. Some studies even question the need for bed rest altogether, claiming that carrying out routine activity right away have superior results in terms of pain relief as well as time off work compared to bed rest [4]. On the other hand, for those who advocate the need of bed rest, the recommended length of resting period in acute low back pain varies from 2 days to 2 weeks. Certain clinicians insist on 3 days of rest as opposed to a longer period based on the lack of difference in pain relief and objective functional improvement of trunk function [5] while others claim a bedrest of up to 2 weeks has merit when patients complain of severe pain [6].

Current recommendations on when to return to work and resume activities of daily living after experiencing acute LDH is largely based on pain intensity. In general, patients are encouraged to return to work with resolving symptoms [7]. While some clinicians institute a specific time frame of 4-6 weeks for resolution before returning to normal activity, the decision is often hinged on the improvement of back pain [8]. Since different individuals differ in the extent and location of disc herniation and the involvement of surrounding structures, the time required for proper healing of affected tissues including annulus fibrosis also differs. Furthermore, each individual has a distinct level of pain threshold, rendering generalization of time frame inadequate. Thus, there is a need for an objective tool to more precisely gauge the recovery process after a disc herniation and the appropriate timing of return to physical activities.

Although segmental motion of lumbar spine has long been used to quantify spinal instability, its investigation in the setting of acute LDH has been overlooked as a quantitative measure of the recovery process. Previous studies have linked sagittal range of motion of spinal segments in the form of functional radiography to recurrence rate and postoperative outcomes. However, to the best of our knowledge, no study has reported its association with pain intensity during the recovery process. Therefore, the purpose of this study was to investigate if the extent of lumbar segmental motion might be correlated with the recovery process in patients with acute single level LDH.

\section{MATERIALS AND METHODS}

From January 2011 to December 2017, a total of 1,215 patients presented with axial low back pain with an onset of 4 weeks or less to the Department of Rehabilitation Medicine at a tertiary medical center either via outpatient clinic or emergency room. After going through proper diagnostic and management process, 514 were found to meet the prerequisite of having both lumbosacral magnetic resonance imaging (MRI) and simple radiograph available for initial radiological assessment. Patients with a pre-existing MRI from another clinic or hospital were also included given that the onset of symptom preceded the date MRI was taken. After excluding 12 patients who decided not to follow up or undergo the prescribed radiographic evaluation, a total of 502 patients with both lumbosacral spine MRI and sequential functional radiographies (flexion and extension lumbar X-ray) in the sagittal plane were included in this study (Fig. 1). Inclusion criteria were as follows: (1) axial low back pain with an onset of less than 4 weeks; (2) a single level disc herniation either at the L4-5 or L5-S1 level confirmed by MRI; and (3) availability of chronological series of sagittal functional radiography throughout the recovery process. Exclusion criteria were: diagnoses of multiple level disc 
Presented with axial low back pain \pm radicular pain with an onset of 4 weeks or less

from January 1, 2011 to December 31, 2017 $(n=1,215)$

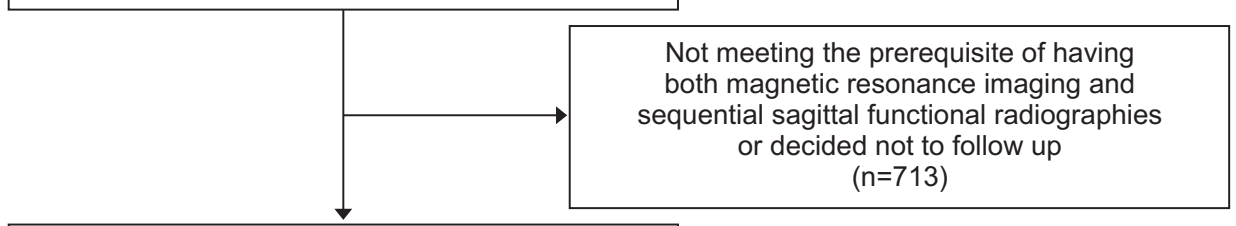

Both lumbosacral magnetic resonance imaging and sequential sagittal functional radiographies available $(n=502)$

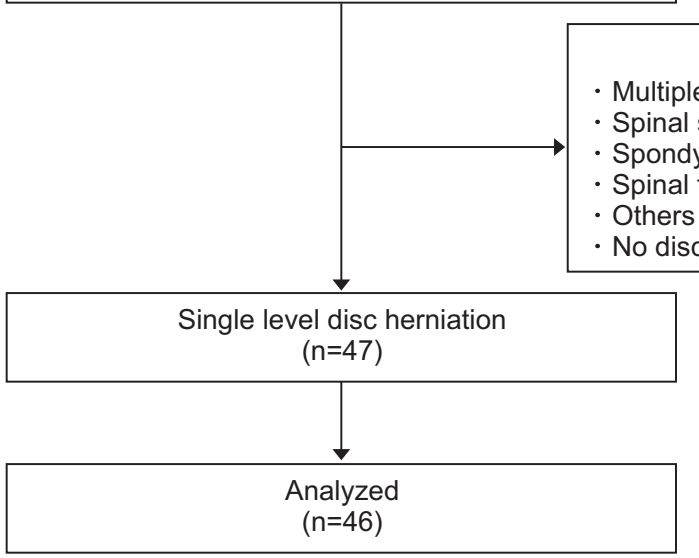

\section{Excluded $(n=455)$}

Multiple level disc disease

Spinal stenosis

Spondyloisthesis

pinal fracture

No disc herniation

$(n=46)$

herniation, spinal stenosis, spondylolisthesis, fracture, or other non-LDH related etiologies.

In clinical assessment, the intensity of pain was evaluated with visual analogue scale (VAS) at the initial presentation. For the present study, patients were only assessed of axial low back pain regardless of the presence of radiating pain to the lower extremities. Pain intensity was documented at every outpatient clinic follow-up. It was evaluated in VAS to monitor disease progression.

Segmental motion of the spine was quantified using a method previously introduced by Kim et al. [9]. Utilizing functional radiographies of lateral flexion and extension lumbar X-ray, segmental range of motion (sROM) of each segment of the lumbosacral spine was measured at the initial evaluation point and subsequent outpatient clinic follow-ups (Fig. 2). Since spinal motion in its entirety could be limited during the acute stage of disc herniation, percentage of segmental range of motion (sROM\%) was also calculated at the aforementioned points to assess contribution made by each particular segment toward lumbar mobility as a whole.

Pearson correlation analysis was performed for clinical and radiologic parameters. Pain intensity in terms of
Fig. 1. A diagram depicting the process of patient selection.
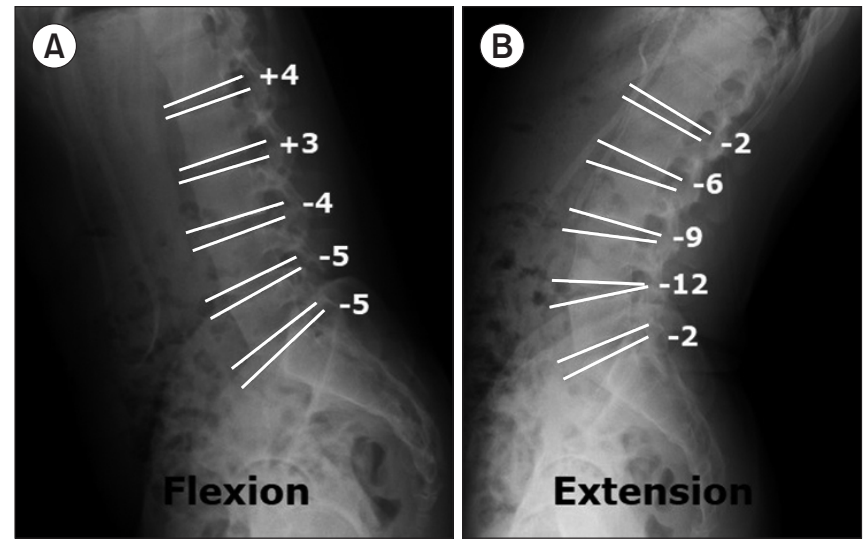

Fig. 2. Method of quantifying sagittal range of motion (sROM) of each lumbosacral segment calculated by first measuring the angle between superior and inferior end plates in flexion (A) and extension (B) radiographs and making subtraction at each respective segment. In this particular patient, sROMs of the five lumbosacral segments L1-2, L2-3, L3-4, L4-5, and L5-S1 were determined to be $6^{\circ}, 9^{\circ}, 5^{\circ}, 7^{\circ}$, and $3^{\circ}$, respectively.

VAS against sROM and sROM\% of each lumbar segment were analyzed at applicable time points. Statistical significance was defined at $p<0.05$. All statistical analyses 
were performed using SPSS software version 18.0 (SPSS Inc., Chicago, IL, USA). The authors have no conflicts of interest to disclose. This study protocol was reviewed and approved by our Institutional Review Board of the aforementioned tertiary hospital (No. KUH1180033).

\section{RESULTS}

Of 502 individuals having both lumbosacral spine MRI and sequential functional radiographies in the sagittal plane, 47 patients were found to have a single level disc herniation at the lumbosacral spine after sorting with exclusion criteria of multiple level disc herniation, spinal stenosis, spondylolisthesis, fracture, and other non-LDH related etiologies. Inspection of the lumbosacral MRI revealed 231 patients with multiple level disc disease, 154 with spinal stenosis, 83 with spondylolisthesis, 51 with spinal fracture, 60 with other etiologies such as SCI and metastasis, and 9 individuals without disc herniation (the sum of each entity amounts to be greater than that of the total number of patients when multiple entities were accounted for).

A total of 46 patients were selected for the final analysis. Classification of disc herniation [10] in this group included 14 protrusions, 30 extrusions, and two cases of sequestration. As for the level of disc herniation, 27 patients had disc herniation at L4-5 level and 19 patients had disc herniation at L5-S1 level. Another patient had single level disc herniation at L2-3 segment. However, since patients were grouped according to the anatomical level for the analysis of sROM and sROM\%, rendering this one patient with disc disease at L2-3 level to be considered for a future study rather than to be included in the current one, in line with the inclusion criteria.

The study group had 26 males and 20 females with a mean age of $41.63 \pm 9.85$ years (Table 1). Age distribution was as follows: 6 patients aged 29 years or less, 18 aged 30-39, 11 aged 40-49, and 11 aged 50 years or older. Onset of low back pain had a mean value of 9.65 days. The number of simple radiographs taken and the time interval in terms of days in between were $2.15 \pm 0.42$ and $41.09 \pm 19.83$, respectively.

The initial pain intensity assessed by VAS had a mean value of 4.61 with a standard deviation of 1.64. Each patient underwent conservative treatment strategies consisting of some combination of life style modifications including bed rest, physical modalities, medications, and epidural steroid injection (ESI) as deemed necessary. Medications encompassed various administration routes, including intravenous injection of methocarbamol, intramuscular injection of diclofenac, oral administration of prednisolone and NSAIDs, and others. Three patients were recommended of surgical treatment at other hospitals. However, each opted out of operation.

Through the recovery process, the pain intensity documented at the final follow-up was improved by a mean VAS of 1.09 with a standard deviation of 1.01 . Of 46 patients, 35 were admitted either via outpatient clinic or emergency room. The duration of admission was $6.50 \pm 3.18$ days while the duration of bedrest was $3.92 \pm 1.93$ days. On the other hand, 11 patients were

Table 1. Patient demographics

\begin{tabular}{|lc}
\hline \multicolumn{1}{c}{ Characteristic } & Value \\
\hline Age (yr) & $41.6 \pm 9.8$ \\
\hline$\leq 29$ & $6(13.0)$ \\
\hline $30-39$ & $18(39.1)$ \\
\hline $40-49$ & $11(23.9)$ \\
\hline$\geq 50$ & $11(23.9)$ \\
\hline Sex & \\
\hline Male & $26(56.5)$ \\
\hline Female & $20(43.5)$ \\
\hline Disc level & \\
\hline L4-5 & $27(58.7)$ \\
\hline L5-S1 & $19(41.3)$ \\
\hline Type of disease entity in MRI findings & \\
\hline Single level LDH & $47(9.4)$ \\
\hline Multiple level LDH & $231(46.0)$ \\
\hline Spinal stenosis & $154(30.7)$ \\
\hline Spondylolisthesis & $83(16.5)$ \\
\hline Fracture & $51(10.2)$ \\
\hline Other & $60(12.0)$ \\
\hline No LDH & $9(1.8)$ \\
\hline MRI not taken & $12(2.4)$ \\
\hline Duration from onset (day) & $9.7 \pm 8.2$ \\
\hline Number of X-rays taken & $2.2 \pm 0.4$ \\
\hline Duration between X-rays (day) & $41.1 \pm 19.8$ \\
\hline Values are presented as mean
\end{tabular}

Values are presented as mean \pm standard deviation or number (\%).

MRI, magnetic resonance imaging; LDH, lumbar disc herniation. 
treated strictly on an outpatient clinic basis, with the mean number of visits of $3.08 \pm 2.23$ times. Overall, the mean treatment duration was 41.09 days with an average of 2.15 X-rays taken. Thirty-two of 46 patients underwent epidural steroid injection procedure, with the number of procedures being $1.24 \pm 0.45$.

Results of analysis for sROM and sROM\% against initial pain intensity in the form of VAS according to the level of disc herniation are shown in Table 2. At the affected segments in both L4-5 and L5-S1 groups, segmental hypomobility was apparent as documented by inverse correlations between VAS and sROM of the affected segment ( $r=-0.83, \mathrm{p}<0.01$ in L4-5; $\mathrm{r}=-0.82, \mathrm{p}<0.05$ in L5-S1) while adjacent segments exhibited decreased segmental motions ( $\mathrm{r}=-0.74, \mathrm{p}<0.05$ in L3-4; $\mathrm{r}=-0.69, \mathrm{p}<0.05$ in L5-S1) in the L4-5 group. As for sROM\%, segmental hypomobility was again present at the affected level in both L4-5 and L5-S1 groups. However, adjacent segments exhibited no statistically significant correlation between VAS and sROM\% in L4-5 disc herniations while other lumbar seg- ments of L1-2 and L2-3 showed increased segmental contribution to lumbar motion as a whole.

As for the change in VAS against sROM and sROM\% (Table 3), the L4-5 group exhibited inverse relationships in both comparisons ( $r=-0.69, \mathrm{p}<0.05$ in $\mathrm{sROM}$; $\mathrm{r}=-0.74$, $\mathrm{p}<0.05$ in sROM\%). In other words, the degree of pain improvement from the initial assessment to the follow-up period correlated with the extent of motional improvement at the affected level in both absolute and relative values quantified as SROM and sROM\%, respectively. In the analysis of L5-S1 group, a similar trend was found in comparisons of VAS against both range of motion parameters $(\mathrm{r}=-0.75, \mathrm{p}<0.05$ in $\mathrm{sROM}$; $\mathrm{r}=-0.78, \mathrm{p}<0.05$ in sROM\%).

\section{DISCUSSION}

A disc herniation occurs when the gelatinous nuclear material ruptures out through a tear in the annulus fibrosus beyond the confinement of the intervertebral disc

Table 2. Relationship of sROM and sROM\% with initial pain intensity in both groups expressed in terms of Pearson correlation coefficient

\begin{tabular}{llllll}
\hline & \multicolumn{4}{c}{ Lain intensity, initial (VAS) } \\
\cline { 2 - 3 } \cline { 5 - 6 } & \multicolumn{2}{c}{ LROM } & \multicolumn{2}{c}{ L5-S1 group } \\
\cline { 2 - 3 } \cline { 5 - 6 } L1-2 & $0.008(\mathrm{p}=0.983)$ & $0.739\left(\mathrm{p}=0.023^{*}\right)$ & & $-0.597(\mathrm{p}=0.211)$ & $0.366(\mathrm{p}=0.475)$ \\
L2-3 & $-0.352(\mathrm{p}=0.352)$ & $0.751\left(\mathrm{p}=0.020^{*}\right)$ & & $-0.712(\mathrm{p}=0.112)$ & $0.593(\mathrm{p}=0.215)$ \\
L3-4 & $-0.738\left(\mathrm{p}=0.023^{*}\right)$ & $-0.489(\mathrm{p}=0.182)$ & & $-0.440(\mathrm{p}=0.383)$ & $0.612(\mathrm{p}=0.197)$ \\
L4-5 & $-0.831\left(\mathrm{p}=0.005^{*}\right)$ & $-0.794\left(\mathrm{p}=0.011^{*}\right)$ & & $-0.540(\mathrm{p}=0.268)$ & $-0.192(\mathrm{p}=0.716)$ \\
L5-S1 & $-0.690\left(\mathrm{p}=0.040^{*}\right)$ & $-0.218(\mathrm{p}=0.574)$ & & $-0.828\left(\mathrm{p}=0.041^{*}\right)$ & $-0.924\left(\mathrm{p}=0.008^{*}\right)$ \\
\hline
\end{tabular}

sROM, segmental range of motion; VAS, visual analogue scale.

${ }^{*} \mathrm{p}<0.05$ was considered significant. Statistical significance test was analyzed by Pearson correlation coefficient.

Table 3. Relationship of changes in sROM and sROM\% with change in pain intensity in both groups expressed in terms of Pearson correlation coefficient

\begin{tabular}{|c|c|c|c|c|}
\hline & \multicolumn{4}{|c|}{ Pain intensity, change (VAS) } \\
\hline & \multicolumn{2}{|c|}{ L4-5 group } & \multicolumn{2}{|c|}{ L5-S1 group } \\
\hline & SROM & sROM\% & sROM & sROM\% \\
\hline $\mathrm{L} 1-2$ & $-0.495(\mathrm{p}=0.176)$ & $0.365(\mathrm{p}=0.334)$ & $-0.672(\mathrm{p}=0.144)$ & $0.153(\mathrm{p}=0.773)$ \\
\hline L2-3 & $-0.545(\mathrm{p}=0.129)$ & $0.557(\mathrm{p}=0.120)$ & $-0.735(\mathrm{p}=0.096)$ & $0.624(\mathrm{p}=0.186)$ \\
\hline L3-4 & $-0.383(\mathrm{p}=0.309)$ & $0.014(\mathrm{p}=0.971)$ & $-0.060(\mathrm{p}=0.910)$ & $0.863(\mathrm{p}=0.057)$ \\
\hline L4-5 & $-0.690(\mathrm{p}=0.039 *)$ & $-0.735\left(\mathrm{p}=0.024^{*}\right)$ & $-0.352(\mathrm{p}=0.493)$ & $0.016(p=0.976)$ \\
\hline L5-S1 & $-0.587(\mathrm{p}=0.096)$ & $-0.045(\mathrm{p}=0.908)$ & $-0.751\left(\mathrm{p}=0.045^{*}\right)$ & $-0.785\left(\mathrm{p}=0.046^{*}\right)$ \\
\hline
\end{tabular}

sROM, segmental range of motion; VAS, visual analogue scale.

${ }^{*} \mathrm{p}<0.05$ was considered significant. Statistical significance test was analyzed by Pearson correlation coefficient. 
space. During this process, the displaced material may stimulate nociceptors in the annulus fibrosus, causing nociceptive pain. Depending on the location and volume of disc material forced out, neural structures might be compressed and irritated, resulting in both mechanical and chemical reactions.

Much analogous to the process of abdominal guarding in acute abdomen which occurs in response to inflammation of the inner abdominal space, the authors believe that, with acute pain in disc herniation, natural immobilization of involved segments takes place. Through this temporary natural fusion for protection, atypical sharing of axial load is materialized with adjacent structures such as facet joints and surrounding muscles. The postulated mechanism involves muscle spasm and increased facet joint fluid, attributing to the protection of the disc material which would be under less axial pressure than usual.

In this sense, the observed hypomobility of the involved segments in acute disc herniation could be explained. The greatest restriction of motion was found at the affected segment. In acute herniation involving the L4-5 disc, our data indicated that the largest decrease in mobility was present at the L4-5 level, with two adjacent segments showing restriction of motion as well, albeit to lesser degrees. As for the L5-S1 disc, the affected segment was once again linked to the greatest motional limitation. However, hypomobility in the adjacent level did not follow the suit. In part, this could be explained by the fact that L5-S1 joint function has a cantilever mechanism [11] where a large portion of force conveyed on the L5-S1 level could be transferred to the sacropelvic complex rather than the adjacent L4-5 segment.

Similarly, assessment of segmental contribution to spinal movement as a whole in the sagittal plane in the form of sROM\% revealed a homologous trend. In both L4-5 and L5-S1 disc herniation groups, the smallest segmental contribution was made by the affected segment toward spinal flexion and extension movements. As for the L4-5 level, non-adjacent segments of the lumbosacral spine, namely L1-2 and L2-3 levels, made greater range of motion with increasing intensity of pain while the same cantilever concept could be applied in the assessment of L5S1 segment.

With the recovery process of disc herniation, the aforementioned restriction on range of spinal motion gradually mitigated with improvement of pain. In both disc level groups, greater alleviation of pain resulted in greater improvement in SROM at the affected level. This was found both in absolute value of SROM and relative contribution toward the whole movement in the form of sROM\%. Therefore, it could be assumed that segmental mobility assessed in terms of sagittal range of motion in the lumbosacral spine could be a useful tool reflecting the recovery process in acute single level disc herniation. In the early stage when pain intensity is at its peak, involved segments exhibit hypomobility in line with transient natural fusion for protection. With resolution of pain comes improvement in the segmental mobility, indicating that overcoming an acute disc herniation could be appropriately documented by examining sagittal range of motion in the lumbosacral spine.

This study has some limitations. First, disc herniation of only a single level was considered for the purpose of delineating correlations between variables in its simplest context. Hence, multi-level disc herniation was excluded. Coincidently, such construction happened to narrow the inclusion window, deducing a less than expected number of patients to be studied. Moreover, since the analysis was performed based on the anatomical level due to the aforementioned difference in biomechanics, exclusion of disc herniation at upper lumbar segments (where sole injury of the particular level is less likely) was inevitable.

Another limitation lies in the retrospective design of this study which could have introduced selection bias and information bias, making it difficult to for exquisitely examine the temporal relationship.

In conclusion, evidently, improvement in sagittal mobility of the affected segment in acute LDH adequately reflected mitigation of low back pain during the recovery process. Therefore, keeping in mind of segmental hypomobility during transient natural fusion, following up on the investigation of SROM in the sagittal plane could serve as a useful tool for clinicians to make recommendations for patients on when to resume premorbid level of physical activities after suffering an episode of acute LDH.

\section{CONFLICT OF INTEREST}

No potential conflict of interest relevant to this article was reported. 


\section{REFERENCES}

1. Vialle LR, Vialle EN, Suarez Henao JE, Giraldo G. Lumbar disc herniation. Rev Bras Ortop 2015;45:17-22.

2. Jordan J, Konstantinou K, O'Dowd J. Herniated lumbar disc. BMJ Clin Evid 2009;2009:1118.

3. Katz JN. Lumbar disc disorders and low-back pain: socioeconomic factors and consequences. J Bone Joint Surg Am 2006;88 Suppl 2:21-4.

4. Malmivaara A, Hakkinen U, Aro T, Heinrichs ML, Koskenniemi L, Kuosma E, et al. The treatment of acute low back pain: bed rest, exercises, or ordinary activity? N Engl J Med 1995;332:351-5.

5. Szpalski M, Hayez JP. How many days of bed rest for acute low back pain? Objective assessment of trunk function. Eur Spine J 1992;1:29-31.

6. Dang L, Liu Z. A review of current treatment for lumbar disc herniation in children and adolescents. Eur Spine J 2010;19:205-14.

7. Atlas SJ, Chang Y, Kammann E, Keller RB, Deyo RA,
Singer DE. Long-term disability and return to work among patients who have a herniated lumbar disc: the effect of disability compensation. J Bone Joint Surg Am 2000;82:4-15.

8. Atlas SJ, Deyo RA. Evaluating and managing acute low back pain in the primary care setting. J Gen Intern Med 2001;16:120-31.

9. Kim KT, Park SW, Kim YB. Disc height and segmental motion as risk factors for recurrent lumbar disc herniation. Spine (Phila Pa 1976) 2009;34:2674-8.

10. Fardon DF, Williams AL, Dohring EJ, Murtagh FR, Gabriel Rothman SL, Sze GK. Lumbar disc nomenclature: version 2.0: recommendations of the combined task forces of the North American Spine Society, the American Society of Spine Radiology and the American Society of Neuroradiology. Spine J 2014;14:252545.

11. Aspden RM. Intra-abdominal pressure and its role in spinal mechanics. Clin Biomech (Bristol, Avon) 1987; 2:168-74. 